\title{
Effective Generation of Milliwatt-Level Sub-Terahertz Wave for Nonlinear Response Measurement of Two-Dimensional Material by Optical Heterodyne Technique
}

\author{
Shuqing Chen, ${ }^{1}$ Zhiqiang Xie, ${ }^{1}$ Junmin Liu, ${ }^{1}$ Yanliang He, ${ }^{1}$ Yao Cai, ${ }^{1}$ \\ Xiaoke Zhang, ${ }^{1}$ Jiangnan Xiao, ${ }^{2}$ Ying Li, $^{1}$ and Dianyuan Fan ${ }^{1}$ \\ ${ }^{1}$ International Collaborative Laboratory of 2D Materials for Optoelectronics Science and Technology and Key Laboratory of \\ Optoelectronic Devices and Systems of Ministry of Education and Guangdong Province, Shenzhen University, Shenzhen 518060, China \\ ${ }^{2}$ Key Laboratory for Information Science of Electromagnetic Waves, Fudan University, Shanghai 200433, China \\ Correspondence should be addressed to Ying Li; queenly@szu.edu.cn
}

Received 19 February 2017; Revised 5 May 2017; Accepted 24 August 2017; Published 22 October 2017

Academic Editor: Jan A. Jung

Copyright (C) 2017 Shuqing Chen et al. This is an open access article distributed under the Creative Commons Attribution License, which permits unrestricted use, distribution, and reproduction in any medium, provided the original work is properly cited.

By using optical heterodyne technique, we demonstrated the stable emission of sub-terahertz wave with the frequency ranging from $88 \mathrm{GHz}$ to $101 \mathrm{GHz}$, which can operate as microwave source for nonlinear response measurement system. Mutual frequency beating of two well-separated sideband signals at a $0.1 \mathrm{THz}$ photo-detector (PD) allows for the generation of sub-terahertz signal. Based on this approach, we have achieved the radiation of $0.1 \mathrm{THz}$ wave with power up to $4 \mathrm{~mW}$. By transmittance measurement, twodimensional nanomaterial topological insulator $\left(\mathrm{TI}: \mathrm{Bi}_{2} \mathrm{Te}_{3}\right)$ shows saturable absorption behaviors with normalized modulation depth of $47 \%$ at $0.1 \mathrm{THz}$. Our results show that optical heterodyne technique could be developed as an effective microwave source generation for nonlinear measurement at sub-terahertz, even terahertz band.

\section{Introduction}

Sub-terahertz (sub-THz) and/or terahertz (THz) wave, usually defined in the range of $0.1-10 \mathrm{THz}$, has been extensively researched owing to its importance for high-frequency wireless communications, radar systems, high resolution imaging applications, and nonlinear measurement systems [1-7]. Especially for areas of measurement domain, the nonlinear characteristic of materials at sub-terahertz band is an influential perspective but was neglected for a long time. As the discovery of graphene, the first two-dimensional Dirac material, its unique band structure gives it ultra-broadband nonlinear response, which shows great prospect in various microwave and optical devices $[8,9]$. To fully explore the nonlinear characteristic of these graphene-like materials and the effects of band structure, the sub-terahertz band which overlapped the microwave and terahertz has stimulated more and more researchers. However, how to investigate the nonlinear response at such high frequency, the high quality and effective microwave source is a real challenge. And for nonlinear excitations, the higher microwave power is particularly important.

It is always highly encouraged to search for a robust method to generate sub-THz/THz wave with advantages of low phase noise, high power, and cost-effective. However, electrical signal generation of high-frequency microwave signals beyond $60 \mathrm{GHz}$ is difficult to be obtained owing to the bandwidth limitations of electronic devices [10, 11]. To fulfill this expectation, photonic generation is considered as a promising technique [12-18]. As an alternative, optical generation, such as optical mode locking $[19,20]$, optical injection locking [21, 22], and external modulation [23-26], has been widely employed to generate $\mathrm{THz}$ wave. In particular, external modulation can provide excellent performance of stability and reliability. But, all those methods involve with very complicated structures and required extra photoelectric devices. By comparison, optical heterodyne technique is regarded as one of the most promising methods for photonics 
generation of sub-THz/THz wave owing to its broad bandwidth, large tunability, efficiency, and cost-effectiveness [2730]. On the other hand, dual-wavelength single-longitudemode fiber ring laser is also demonstrated to generate highfrequency microwave $[31,32]$, but the output performance is not very stable and a complex dual-wavelength fiber ring laser needs to be designed. And difference frequency mixing of two collinearly propagating optical beams inside electro-optic crystals can be used to generate $\mathrm{THz}$ signals [33]. However, all-fiber format is lost and its application in miniaturization and integration is limited. By using the optical heterodyne technique, Tang et al. have realized the $100 \mathrm{GHz}$ microwave generation and $2 \mathrm{~m}$ wireless transmission [34]. But, it is also necessary to further study the performance of the generated sub-terahertz, especially the radiation power, and stability shows importance in terahertz measurement, and its practical application in radio-over-fiber technique also should be paid more attention.

In this paper, we experimentally demonstrated an effective approach for sub-THz/THz wave generation by optical heterodyne technique. Two distributed feedback (DFB) lasers were employed to generate optical microwave signal, with frequency ranging from $88 \mathrm{GHz}$ to $101 \mathrm{GHz}$. The output power directly radiated towards free space reached up to $4 \mathrm{~mW}$. And it was found experimentally that fiber dispersion contributed much less effect to the radiation power. Based on this sub-terahertz source, the transmittance experiment system was constructed and used to study the nonlinear absorption characteristic of two-dimensional Dirac material (TI: $\mathrm{Bi}_{2} \mathrm{Te}_{3}$ nanoplatelets, TI NPs). The experiment results reveal the saturable absorption behaviors of the TI: $\mathrm{Bi}_{2} \mathrm{Te}_{3}$, with normalized modulation depth of $47 \%$ and saturable intensity of $32 \mu \mathrm{W} / \mathrm{cm}^{2}$. To further evaluate the quality, the microwave source integrated radio-over-fiber (ROF) communication system was under our investigation. In the communication system, we realized $6 \mathrm{~Gb} / \mathrm{s}$ OOK signal transmission over $5 \mathrm{~m}$ wireless link. This illustrated a central point that the generated microwave source has a better performance of quality and stability, which is very critical for the measurement system. These results showed that the generated sub-THz has sufficiently high power and excellent beam quality that may fit for nonlinear response measurement and wireless communication.

\section{Experiment and Results}

2.1. High Power 0.1 THz Sub-Terahertz Wave Generation. The principle of mm-wave generating and phase controlling is shown in Figure 1. The CW light wave, modulated RF signal through a Mach-Zehnder modulator (MZM), is used to generate optical sidebands for optical mm-wave carrier generating. Through an optical comb filter, two second-order optical sidebands are separated out, and the phase information is loaded to one of them by phase modulator. And then, the sideband carried with phase information is recombined with the optical mm-wave to realize the phase controlling of mm-wave. A photodetector (PD) is employed to detect the optical signal realizing mm-wave signal conversion [31].
The experimental setup of $0.1 \mathrm{THz}$ wave generation is illustrated in Figure 1. Two individual continuous light beams came from the DFBs with wavelength fixed at $1546.082 \mathrm{~nm}$ and $1546.858 \mathrm{~nm}$, respectively. Then, they were recombined and directed into a 50/50 optical coupler (OC), from which dual-wavelength lasing spectrum had been measured as shown in the insert of Figure 1. In the following, an optical attenuator was used to adjust the input optical power before the PD, which can convert the optical signals to the sub$\mathrm{THz}$ microwave signal through the mutual frequency beating effect. The generated $0.1 \mathrm{THz}$ wave was further amplified by a high-frequency electrical amplifier and radiated to free space by a horn antenna (gain: $25 \mathrm{dBi}$ ). Before detecting the $0.1 \mathrm{THz}$ wave by the absolute $\mathrm{THz}$ power meter, a chopper was employed to modulate it with a fixed frequency of $30 \mathrm{MHz}$.

The stability of the generated sub-THz wave has been studied. At an input optical power of $3.6 \mathrm{~mW}$, the radiated power of sub-THz wave was measured to be about $3.2 \mathrm{~mW}$. By repeatedly monitoring the radiation power, we note that the power was almost kept constant over 10 minutes at room temperature, as shown in Figure 2, indicating the high stability of the radiation output. If one input wavelength was fixed at $1546.858 \mathrm{~nm}$, sub-THz radiation at different frequencies can be produced as the other wavelength continuously changed. Experimentally, the output frequencies of the wave can be tuned from $88 \mathrm{GHz}$ to $101 \mathrm{GHz}$, while the input optical power was kept at $3.6 \mathrm{~mW}$. Owing to the bandwidth limitation of the amplifier, we note that the operating frequency has a dynamic range and the optimal operating frequency located from $96 \mathrm{GHz}$ to $98 \mathrm{GHz}$, as shown in Figure 3(a). Particularly, the microwave power decreased dramatically for the frequency beyond $99 \mathrm{GHz}$, as the input optical power holding constant.

Figure 3(b) demonstrates the relationship of input optical power and radiation microwave power at different situations. Under the weak power regime, the output power almost synchronously increased with the increase of the input power. However, once the input power exceeds $6 \mathrm{~mW}$, the output power becomes saturated. In order to study the role of the fiber dispersion on the microwave generation, transmission experiments through back to back (BTB), $10 \mathrm{~km}$ DSF, $10 \mathrm{~km}$ single mode fiber (SMF), and $20 \mathrm{~km}$ SMF were under investigation. However, as noted in Figure 3(b), there is no significant difference, indicating that the real impact of fiber dispersion is limited.

2.2. Nonlinear Response Measurement of TI at Sub-Terahertz $B$ and. A quality and stable microwave source is essential for nonlinear response measurement of variety nonlinear media, especially for sub-terahertz which overlapped the microwave and terahertz band. The microwave generated by optical heterodyne technique has many great assets in nonlinear response measurement, such as broadband tenability of wavelength and high output power. To verify its application in nonlinear response measurement, we constructed the measurement system like that in $[8,26]$ to investigate the nonlinear absorption property of the TI (TI: $\mathrm{Bi}_{2} \mathrm{Te}_{3} \mathrm{NPs}$ transferred onto the square quartz glass) [26].

Here, the diethylene glycol (DEG) mediated polyol method was introduced into the composite few-layer TI: 


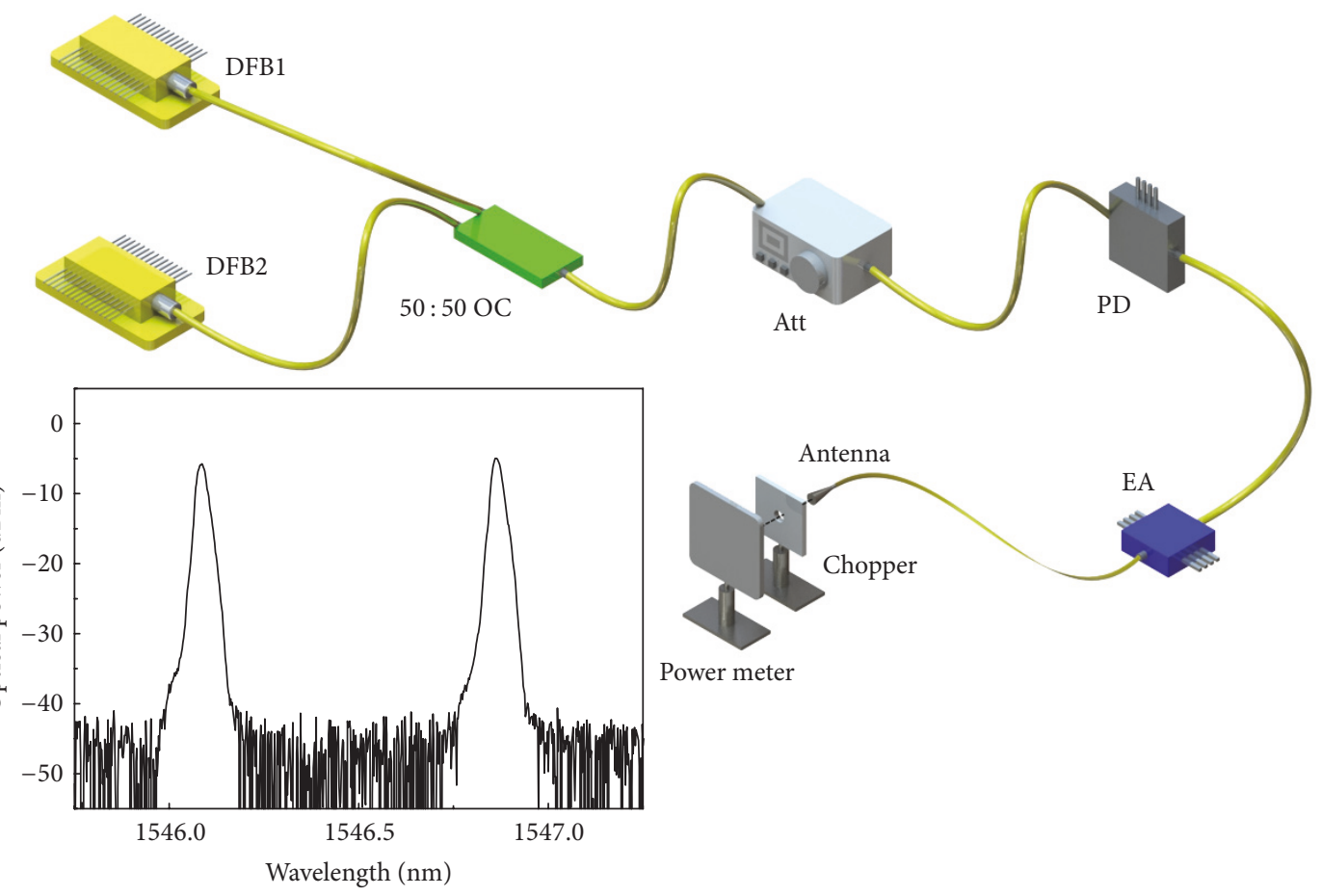

Figure 1: Schematic of the $0.1 \mathrm{THz}$ microwave generation system, and the insert is the optical spectrum of the source for optical heterodyne.

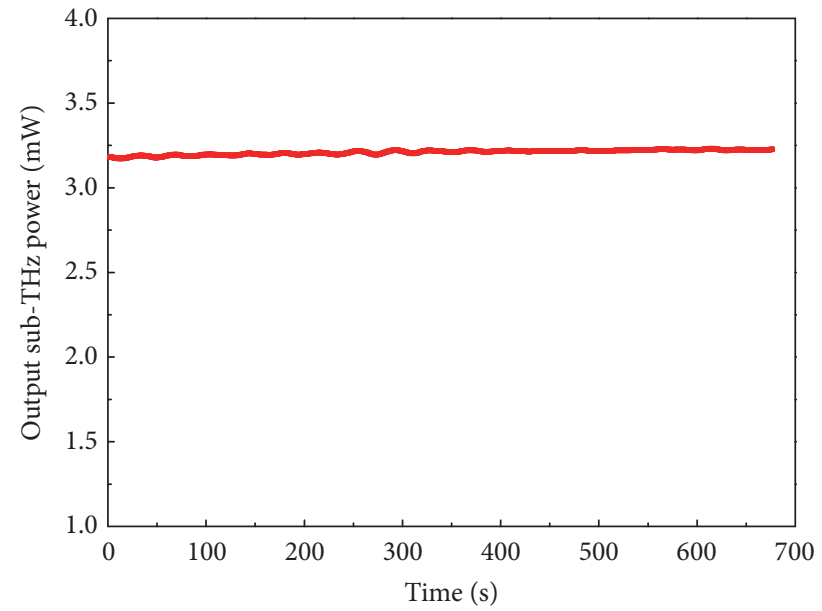

FIGURE 2: Power radiated from the antenna takes over 10 minutes with input optical power at $3.6 \mathrm{~mW}$.

$\mathrm{Bi}_{2} \mathrm{Te}_{3}$ NPs [35]. The physical photo of $\mathrm{TI}: \mathrm{Bi}_{2} \mathrm{Te}_{3}$ ethanol solution is shown in Figure 4(a). Figure 4(b) shows the transmission electron microscopy (TEM) images; it can be clearly seen that TI exhibits symmetric hexagonal morphology, which indicates relative high stability [26]. Atomic force microscope (AFM) images are shown in Figure 4(c), it further corroborated the symmetric hexagonal morphology of prepared TI NPs, and the sample thickness is measured to be an average of $55 \mathrm{~nm}$.

The experimental setup shows in Figure 5(a) that, with the attenuator adjusting, the microwave power can be changed from $20 \mu \mathrm{W}$ to $4 \mathrm{~mW}$. The chopper has the aperture diameter of $15 \mathrm{~mm}$, which shows that the maximum output intensity can reach $2.27 \mathrm{~mW} / \mathrm{cm}^{2}$. This is a sufficient approach for most measurement. The corresponding transmittance curve at this sub-terahertz band is shown in Figure 5(b); as can be seen from the diagram, the transmittance goes to a steady value with the input intensity increasing from 20 to $160 \mu \mathrm{W} / \mathrm{cm}^{2}$. After fitting with formula, $T(I)=1-\Delta T * \exp \left(-I / T_{\text {sat }}\right)-$ $T_{\text {ns }}$, where $T(I)$ is the transmission, $\Delta T$ is the modulation depth, $I$ is the input intensity, $I_{\text {sat }}$ is the saturation power intensity, and $T_{\mathrm{ns}}$ is the nonsaturable absorbance, we obtained the corresponding saturable absorption parameters [36]. The modulation depth and saturation intensity of TI are $47 \%$ and $32 \mu \mathrm{W} / \mathrm{cm}^{2}$, respectively. The results coincide with the results reported in [26], which indicated that the sub-terahertz result from this approach is of high quality and appropriate for nonlinear response measurement.

As schematically shown in Figure 6(a), the structure of TI: $\mathrm{Bi}_{2} \mathrm{Te}_{3}$ can be considered as layers made up of 5 atom thick Te-Bi-Te-Bi-Te covalently bound sheets coupled together by much weaker van der Waals forces [35]. And its band structure is shown in Figure 6(b); like graphene, they are all direct-band-gap material, which is conductive to exciting electronic transitions. Except for the difference that $\mathrm{TI}: \mathrm{Bi}_{2} \mathrm{Te}_{3}$ has insulating bulk state, it also possesses the gapless metallic surface state like graphene. This raises an interesting issue about what role did the insulating bulk state and metallic surface state play in the nonlinear response. As the $\mathrm{TI}: \mathrm{Bi}_{2} \mathrm{Te}_{3}$ was on exposure to light or microwave, the electrons in the valence band can be excited to conduction band and occupied the lowest energy states following 


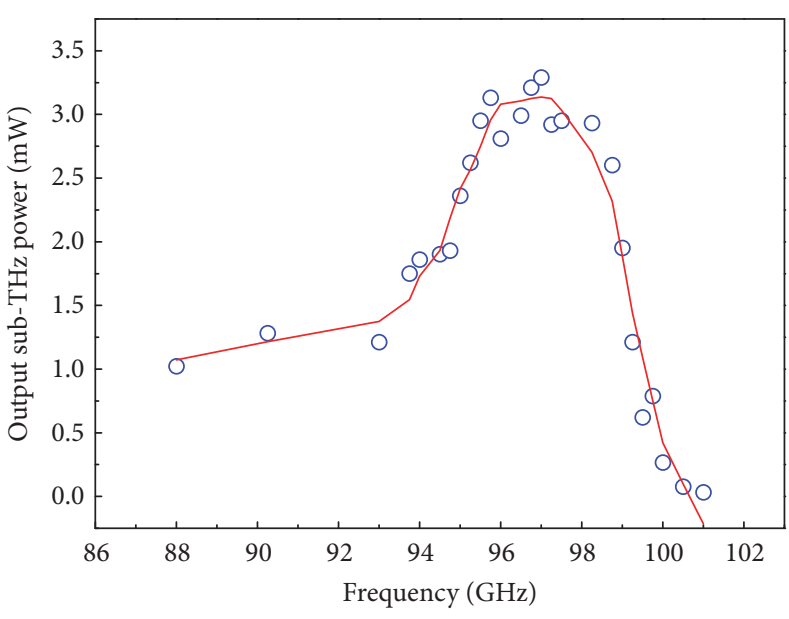

(a)

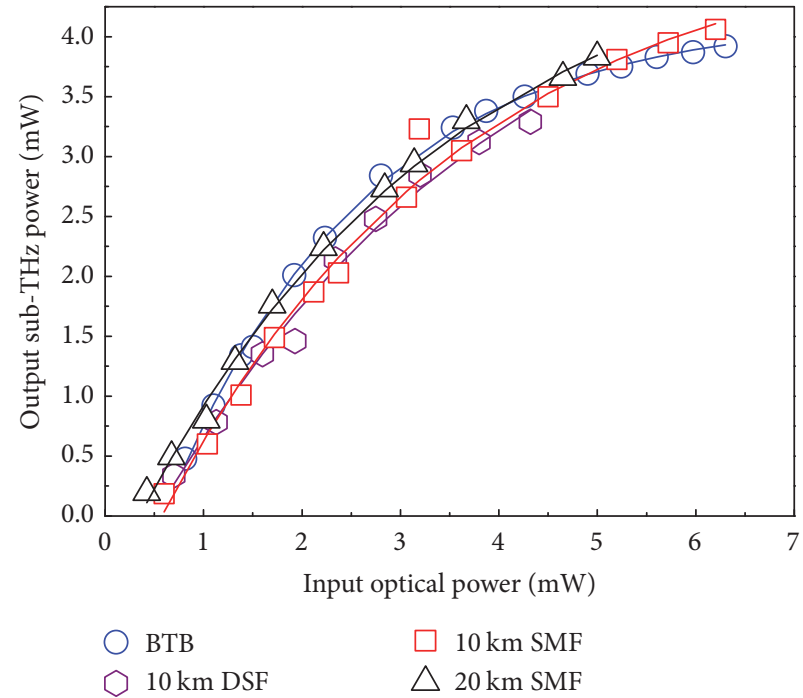

(b)

FIGURE 3: Dependence of sub-THz wave radiation power (a) on different wavelength spacing with input optical power at $3.6 \mathrm{~mW}$ and (b) on PD input optical power with BTB, $10 \mathrm{~km}$ DSF, $10 \mathrm{~km}$ SMF, and $20 \mathrm{~km}$ SMF.

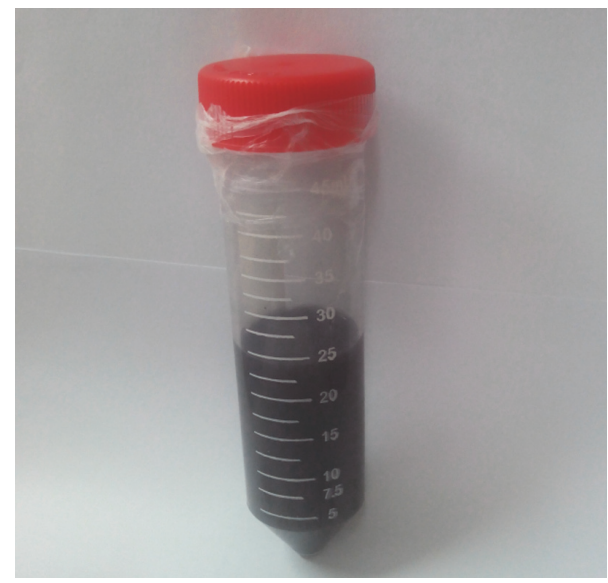

(a)

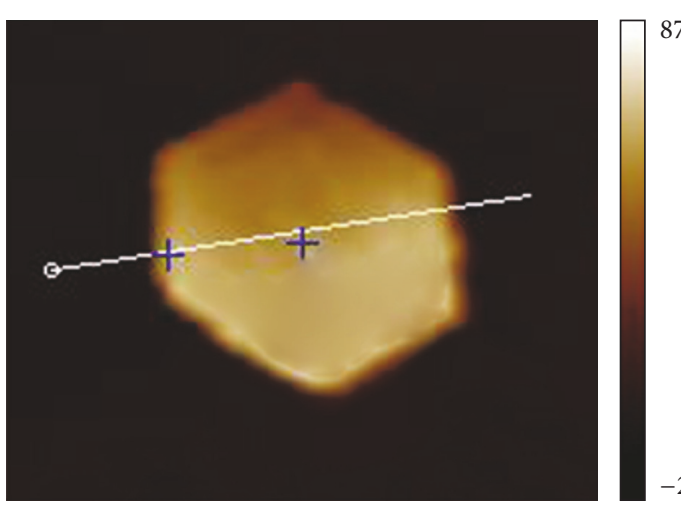

(c)

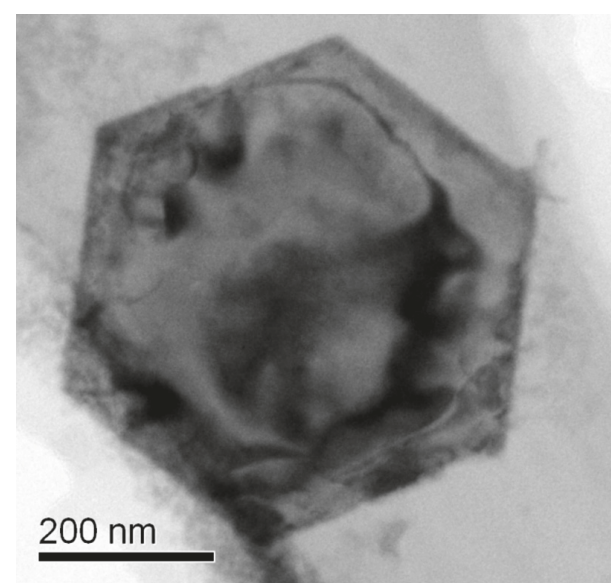

(b)

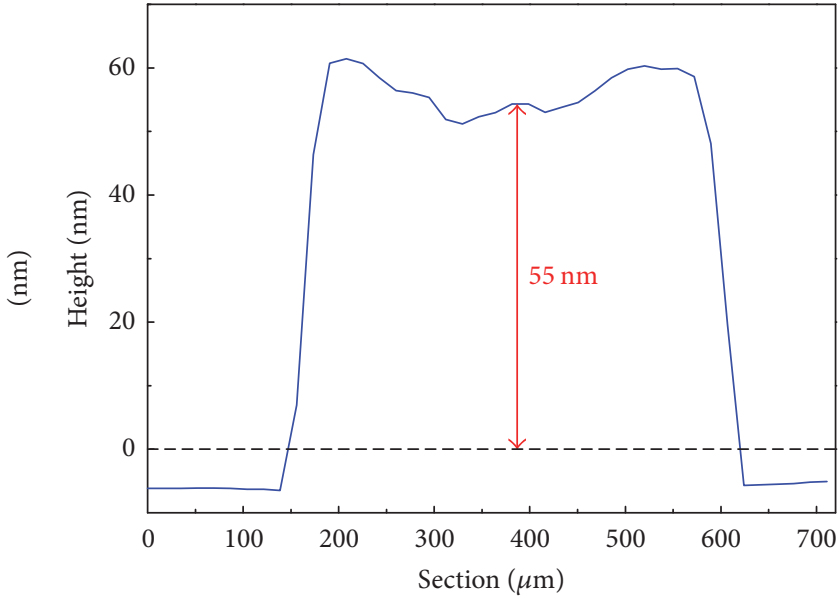

(d)

Figure 4: (a) The physical photo of TI: $\mathrm{Bi}_{2} \mathrm{Te}_{3}$ ethanol solution, (b) TEM images, (c) AFM images, and height profiles of the as-prepared few-layer TI: $\mathrm{Bi}_{2} \mathrm{Te}_{3} \mathrm{NPs}$. 


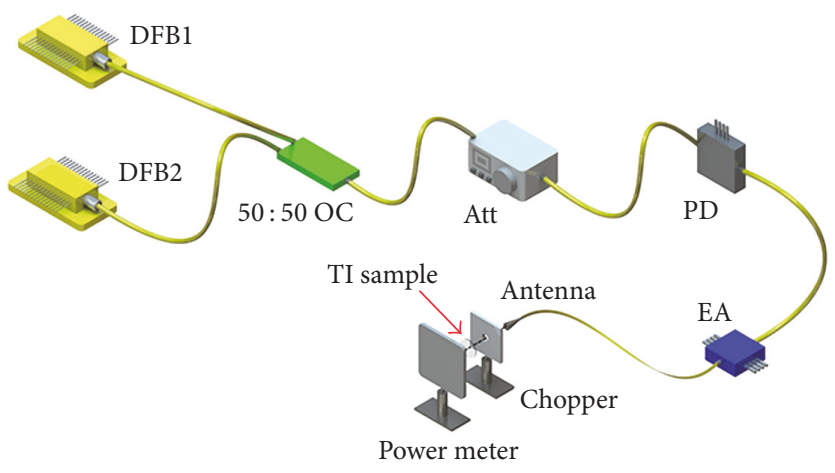

(a)

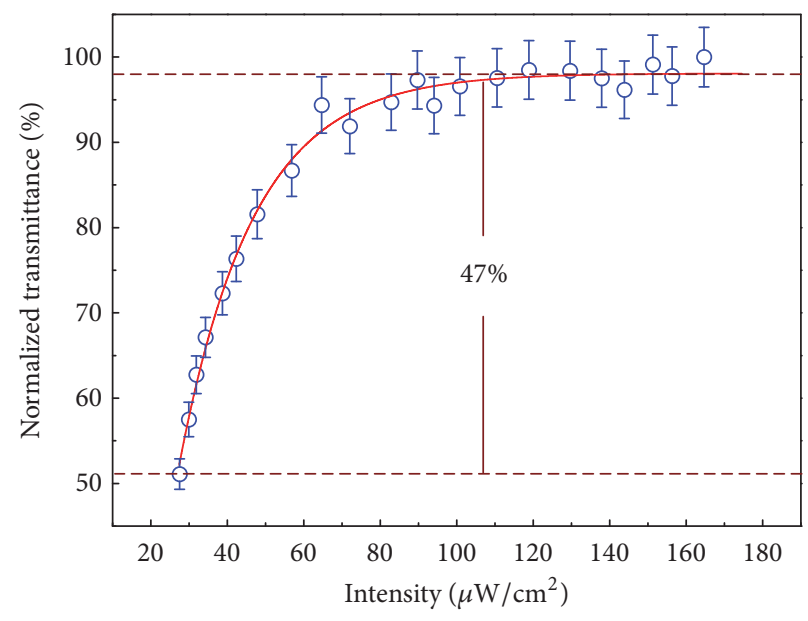

(b)

FIGURE 5: (a) Experimental setup for nonlinear response measurement system and (b) the measured normalized transmittance curve of TI: $\mathrm{Bi}_{2} \mathrm{Te}_{3}$ at sub-terahertz band.

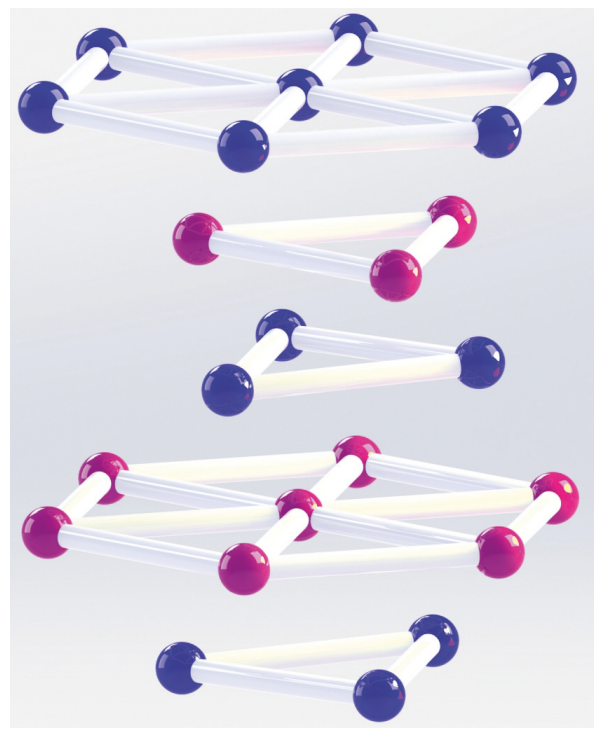

$\Leftrightarrow \mathrm{Pe}$

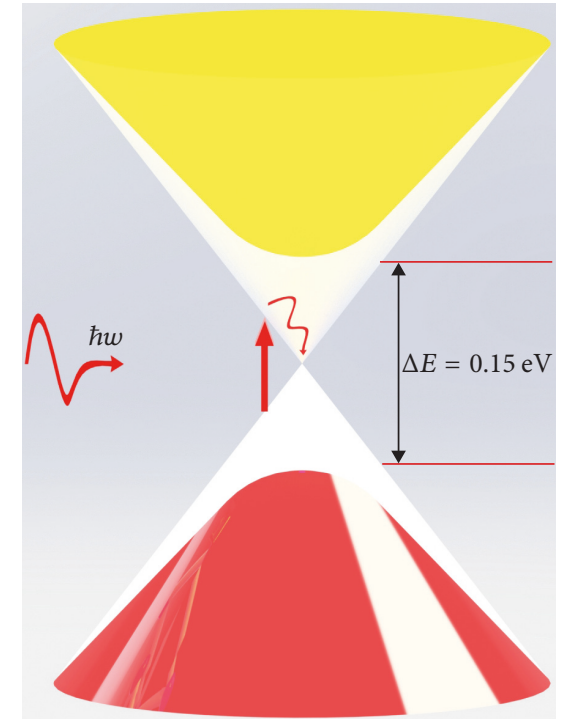

(b)

Figure 6: (a) The atomic structure and (b) the band structure of TI: $\mathrm{Bi}_{2} \mathrm{Te}_{3}$.

the Pauli exclusion principle. With the incident intensity increasing, the generated carriers fill the valence bands and prevent the further excitation of electrons at valance band leading to saturated. However, the insulating band-gap value is about $0.15 \mathrm{eV}$ (at the bulk state of TI: $\mathrm{Bi}_{2} \mathrm{Te}_{3}$ ), indicating that the single photon energy less than $0.15 \mathrm{eV}$ is difficult to excite the electron leaps into conduction band at the bulk state. This saturable absorption process at sub-terahertz band confirmed that the surface metallic state is responsible for the saturable absorption at microwave band, where the single photon energy is far below $0.15 \mathrm{eV}$. All this suggests that the microwave/terahertz band with low photo energy may has important significance in more detailed study of the linear/nonlinear response of material. And the effective microwave source is a guarantee of the linear/nonlinear measurement.

2.3. Radio-over-Fiber Communication System with $0.1 \mathrm{THz}$ Sub-Terahertz Wave. To further evaluate the quality of the generated sub-terahertz wave, it was used to carry digital 


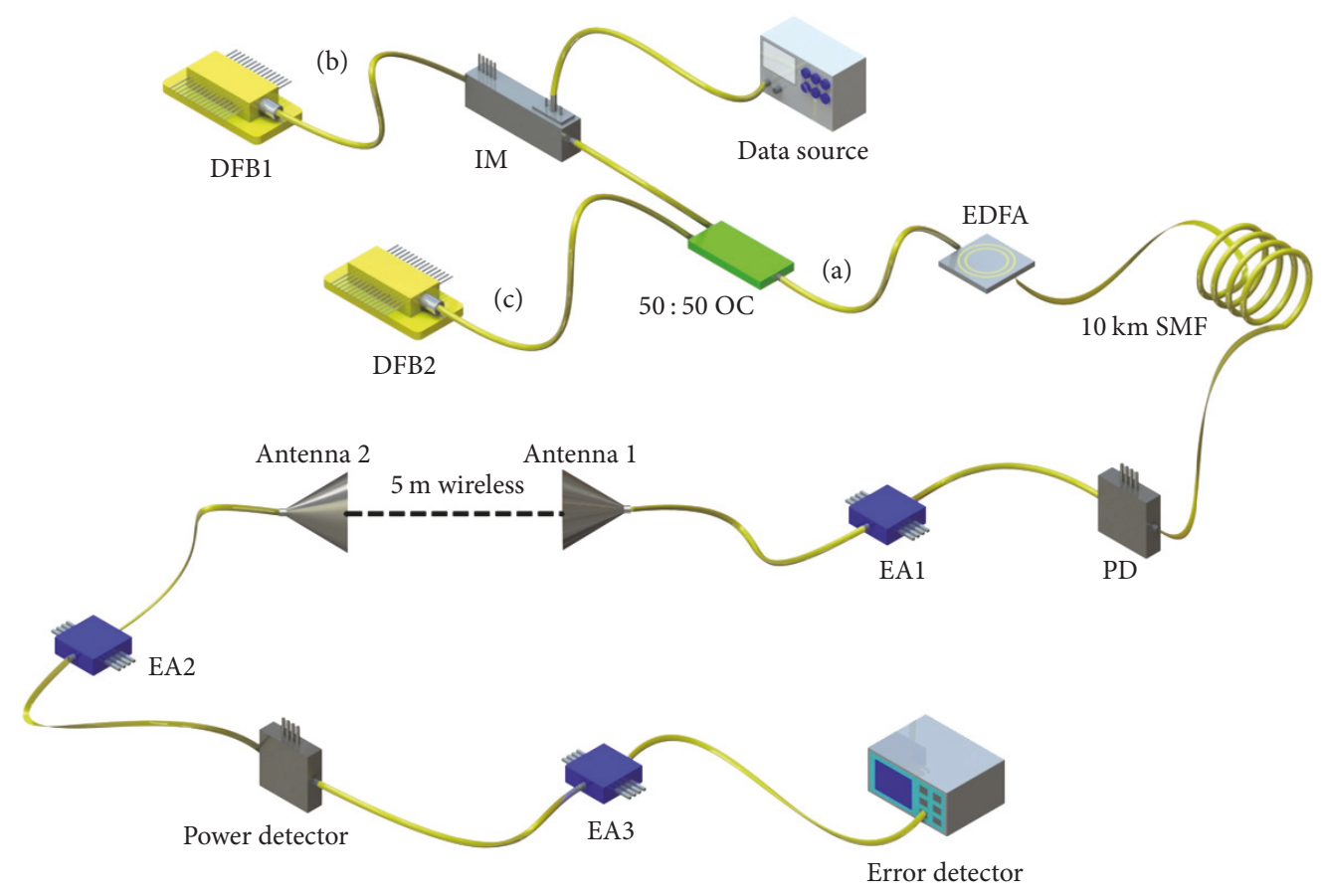

FIGURE 7: Experimental setup of radio-over-fiber system with $0.1 \mathrm{THz}$ carrier.

signal. Figure 7 shows the experimental setup of $0.1 \mathrm{THz}$ radio-over-fiber system with $5 \mathrm{~m}$ delivery. The continuouswave $(\mathrm{CW})$ at $1546.082 \mathrm{~nm}$ and $1546.858 \mathrm{~nm}$ with $\sim 6 \mathrm{dBm}$ output power emitted from DFBs functions as the optical source, with the corresponding optical spectrum shown in Figures 8(b) and 8(c), respectively. This suggests that the frequency spacing between DFB1 and DFB2 is $97 \mathrm{GHz}$. And the CW light wave at $1546.082 \mathrm{~nm}$ from DFB1 was modulated by intensity modulator (IM). The $2 \mathrm{~Gb} / \mathrm{s}$ downlink baseband signals with a pseudorandom binary sequence (PRBS) length of 231-1 were used to drive the IM. Together with the output of DFB2, both of them acted as input of the 50/50 OC, with the optical spectrum shown in Figure 8(a). After $10 \mathrm{~km}$ SMF-28 transmission, in order to obtain the optimal output, which requires that the input power into the $\mathrm{PD}$ cannot be too low, a following erbium-doped fiber amplifier (EDFA) was utilized to compensate the attenuation of the fiber. Then a $0.1 \mathrm{THz}$ PD was employed to detect the optical signal and therefore allows for the conversion of the $0.1 \mathrm{THz}$ signals. The signals were subsequently amplified by a low-noise electrical amplifier (EA1). And then a W-band antenna with a gain of $25 \mathrm{dBi}$ was used to radiate the $0.1 \mathrm{THz}$ wave with $2 \mathrm{~Gb} / \mathrm{s}$ OOK signals loaded.

After $5 \mathrm{~m}$ wireless delivery, the data were received by another antenna with identical parameters of antenna 1 and were then amplified by EA2. Then, a power detector was used to downconvert the data into the baseband. After a broadband EA3, signals were launched into an error detector to measure the BER index.

The BER performances of the data signals under different conditions are shown in Figure 6. The insert (a) of Figure 9 is the eye diagram after only $5 \mathrm{~m}$ wireless transmission, while the insert (b) shows the eye diagram after $10 \mathrm{~km}$ SMF-28 and $5 \mathrm{~m}$ wireless transmission. The result shows that the presence of fiber in the system can significantly distort the eye diagram, which is caused by fiber dispersion [23]. And the power penalty was measured to be $3 \mathrm{~dB}$, as shown in Figure 9.

When the data were loaded at different rates, the corresponding eye diagrams after $5 \mathrm{~m}$ wireless transmission were also measured. As shown in Figure 10, Figures 10(a)-10(d) are the eye diagram as data rate sets at $3,4,5$, and $6 \mathrm{~Gb} / \mathrm{s}$, respectively. The results show that once the loading data rate was set beyond $5 \mathrm{~Gb} / \mathrm{s}$, significant distortion can occur. This is because of bandwidth limitation of the electrical amplifiers. By upgrading the amplifiers, the output power and transmission distance can be scaled up.

\section{Conclusion}

In conclusion, we had experimentally demonstrated a robust method for generating $0.1 \mathrm{THz}$ signal based on optical heterodyne technique, together with its application for nonlinear response measurement. The frequency of radiation microwave was confined at the range of $0.1 \mathrm{THz}$ due to the limitation of electrical amplifier. Further long distance transmission experiments indicate that chromatic dispersion shows limited effect on the radiation power, and $6 \mathrm{~Gb} / \mathrm{s}$ OOK signals were successfully propagated across $5 \mathrm{~m}$ wireless carried by the $0.1 \mathrm{THz}$ wave indicating the generated subterahertz wave is of high quality. Under the $0.1 \mathrm{THz}$ subterahertz wave excitation, TI shows saturable absorption behaviors which further corroborated the contribution of the metallic surface state of $\mathrm{TI}: \mathrm{Bi}_{2} \mathrm{Te}_{3}$ in nonlinear absorption response. Our result constitutes a major step forward the 


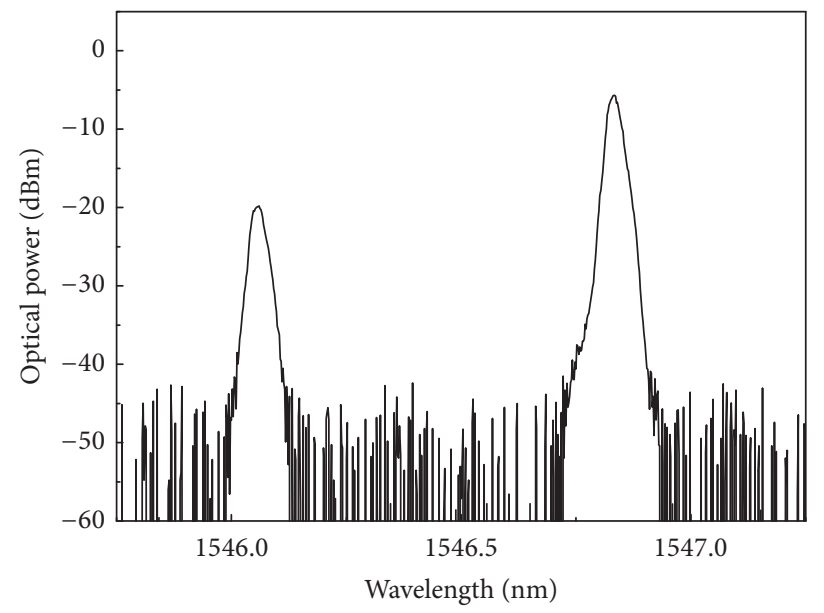

(a)

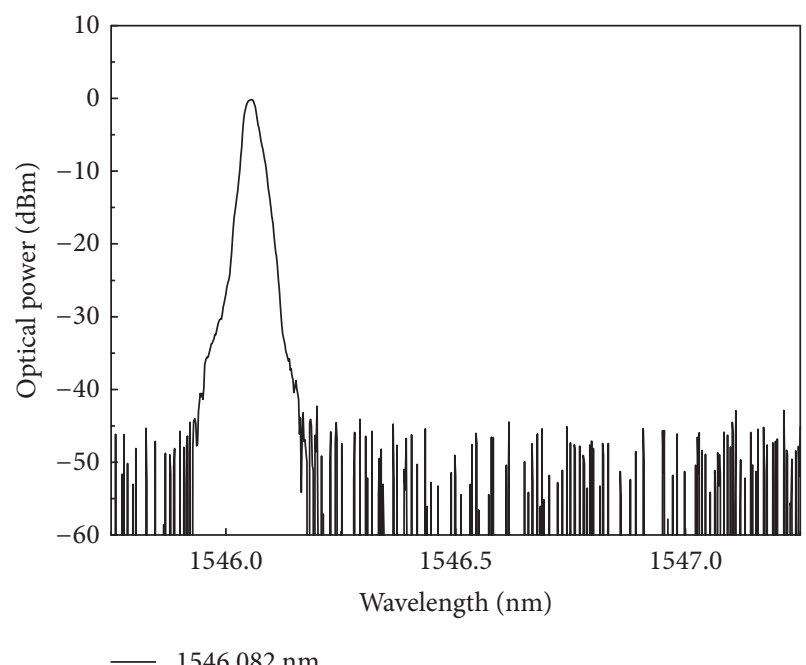

(b)

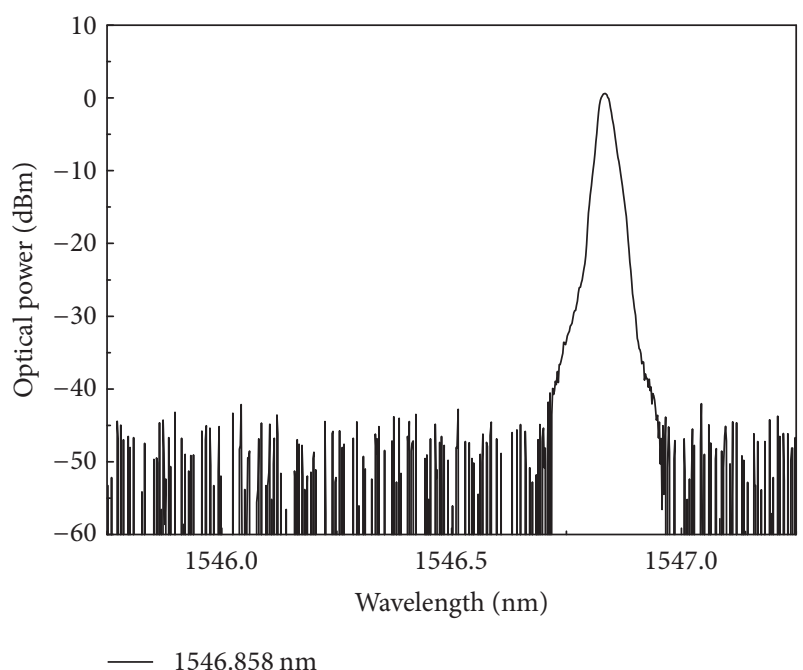

(c)

FIGURE 8: Optical spectrum (a) after OC, (b) after DFB1, and (c) after DFB2, as shown in Figure 7.

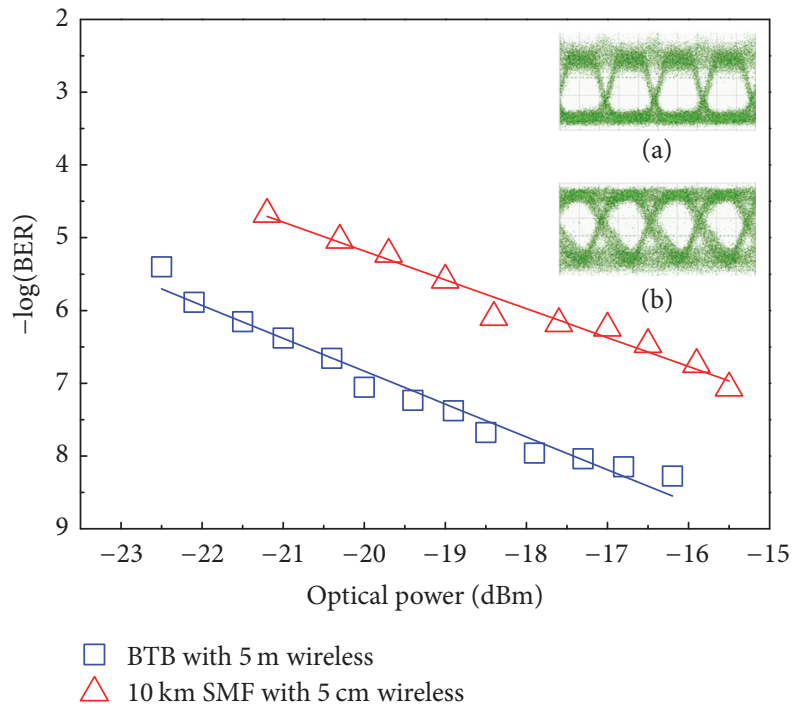

FIGURE 9: The measured BER curves of $2 \mathrm{~Gb} / \mathrm{s}$ signal, insert eye diagrams of signals (a) after $5 \mathrm{~m}$ wireless transmission without fiber, and (b) after $10 \mathrm{~km}$ fiber and $5 \mathrm{~m}$ wireless. 


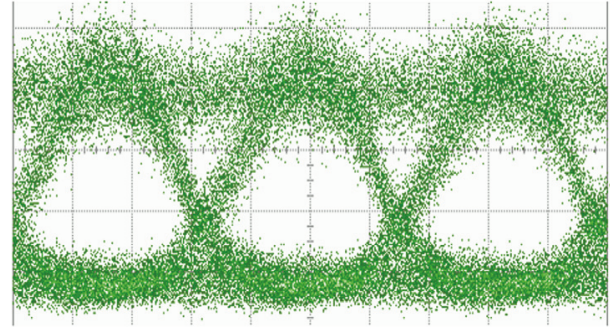

(a)

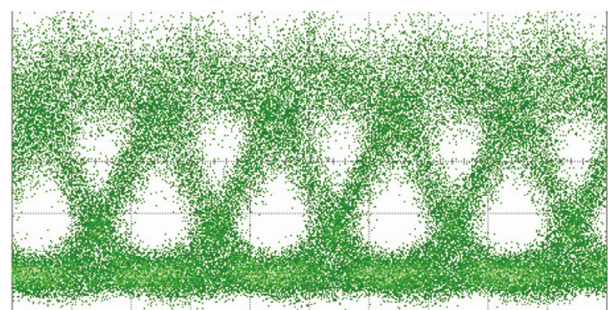

(c)

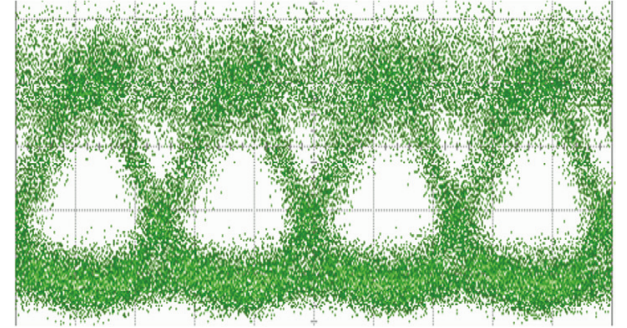

(b)

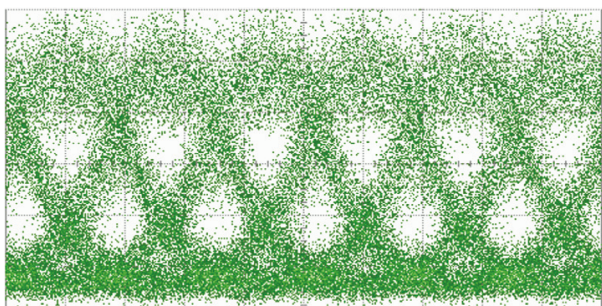

(d)

FIGURE 10: Eye diagram after $5 \mathrm{~m}$ wireless transmission when data rates are (a) 3, (b) 4, (c) 5, and (d) $6 \mathrm{~Gb} / \mathrm{s}$.

development of photonic generation of high-power subterahertz and terahertz source, which shows tremendous potential in measurement domain.

\section{Conflicts of Interest}

The authors declare that they have no conflicts of interest.

\section{Acknowledgments}

This work is partially supported by the program of Fundamental Research of Shenzhen Science and Technology Plan (Grants nos. JCYJ20160422152152634, JCYJ2016032814464, and JCYJ20150324141711651), the National Science Foundation of China (Grants nos. 61575127 and 61505122), the Project Supported by Guangdong Natural Science Foundation (Grant nos. 2016A030310065 and 2014A030310279), the Natural Science Foundation of SZU (Grant nos. 000059 and 2016031), Science and Technology Planning Project of Guangdong Province (2016B050501005), and the Natural Science Foundation Guangdong Education Department (Grants nos. 2015KTSCX124 and 2015KQNCX146).

\section{References}

[1] T. Nagatsuma, "Generating Millimeter and Terahertz Waves," IEEE Microwave Magazine, vol. 10, no. 4, pp. 64-74, 2009.

[2] M. Tonouchi, "Cutting-edge terahertz technology," Nature Photonics, vol. 1, no. 2, pp. 97-105, 2007.

[3] I. Duling and D. Zimdars, "Terahertz imaging: Revealing hidden defects," Nature Photonics, vol. 3, no. 11, pp. 630-632, 2009.

[4] H.-J. Song and T. Nagatsuma, "Present and future of terahertz communications," IEEE Transactions on Terahertz Science and Technology, vol. 1, no. 1, pp. 256-263, 2011.
[5] S. J. B. Yoo, R. P. Scott, D. J. Geisler, N. K. Fontaine, and F. M. Soares, "Terahertz information and signal processing by RF-photonics," IEEE Transactions on Terahertz Science and Technology, vol. 2, no. 2, pp. 167-176, 2012.

[6] A. Hirata, M. Harada, and T. Nagatsuma, "120-GHz Wireless Link Using Photonic Techniques for Generation, Modulation, and Emission of Millimeter-Wave Signals," Journal of Lightwave Technology, vol. 21, no. 10, pp. 2145-2153, 2003.

[7] D. M. Mittleman, R. H. Jacobsen, and M. C. Nuss, “T-ray imaging," IEEE Journal on Selected Topics in Quantum Electronics, vol. 2, no. 3, pp. 679-692, 1996.

[8] Z. Zheng, C. Zhao, S. Lu et al., "Microwave and optical saturable absorption in graphene," Optics Express, vol. 20, no. 21, pp. 23201-23214, 2012.

[9] V. P. Gusynin, S. G. Sharapov, and J. P. Carbotte, "Unusual microwave response of dirac quasiparticles in graphene," Physical Review Letters, vol. 96, no. 25, Article ID 256802, 2006.

[10] H.-W. Hübers, “Terahertz heterodyne receivers," IEEE Journal on Selected Topics in Quantum Electronics, vol. 14, no. 2, pp. 378391, 2008.

[11] H.-J. Song, N. Shimizu, and T. Nagatsuma, "Generation of twomode optical signals with broadband frequency tunability and low spurious signal level," Optics Express, vol. 15, no. 22, pp. 14901-14906, 2007.

[12] E. Rouvalis, M. J. Fice, C. C. Renaud, and A. J. Seeds, "Millimeter-wave optoelectronic mixers based on uni-traveling carrier photodiodes," IEEE Transactions on Microwave Theory and Techniques, vol. 60, no. 3, pp. 686-691, 2012.

[13] H. Ito, T. Furuta, F. Nakajima, K. Yoshino, and T. Ishibashi, "Photonic generation of continuous $\mathrm{THz}$ wave using unitraveling-carrier photodiode," Journal of Lightwave Technology, vol. 23, no. 12, pp. 4016-4021, 2005.

[14] D. Saeedkia and S. Safavi-Naeini, "Terahertz photonics: Optoelectronic techniques for generation and detection of Terahertz waves," Journal of Lightwave Technology, vol. 26, no. 15, pp. 2409-2423, 2008. 
[15] W. Liu, M. Wang, and J. Yao, "Tunable microwave and subterahertz generation based on frequency quadrupling using a single polarization modulator," Journal of Lightwave Technology, vol. 31, no. 10, Article ID 6487368, pp. 1636-1644, 2013.

[16] D. Stanze, A. Deninger, A. Roggenbuck, S. Schindler, M. Schlak, and B. Sartorius, "Compact cw terahertz spectrometer pumped at $1.5 \mu \mathrm{m}$ wavelength," Journal of Infrared, Millimeter, and Terahertz Waves, vol. 32, no. 2, pp. 225-232, 2011.

[17] J. Ye, L. Yan, Z. Chen et al., "Photonic generation of microwave phase-coded signals based on frequency-to-time conversion," IEEE Photonics Technology Letters, vol. 24, no. 17, pp. 1527-1529, 2012.

[18] J. Ye, L. Yan, W. Pan et al., "Photonic generation of triangularshaped pulses based on frequency-to-time conversion," Optics Letters, vol. 36, no. 8, pp. 1458-1460, 2011.

[19] Z. Deng and J. Yao, "Photonic generation of microwave signal using a rational harmonic mode-locked fiber ring laser," IEEE Transactions on Microwave Theory and Techniques, vol. 54, no. 2, pp. 763-767, 2006.

[20] A. C. Bordonalli, B. Cai, A. J. Seeds, and P. J. Williams, "Generation of microwave signals by active mode locking in a gain bandwidth restricted laser structure," IEEE Photonics Technology Letters, vol. 8, no. 1, pp. 151-153, 1996.

[21] L. Goldberg, H. F. Taylor, J. F. Weller, and D. M. Bloom, "Microwave signal generation with injection-locked laser diodes," Electronics Letters, vol. 19, no. 13, pp. 491-493, 1983.

[22] L. Goldberg, A. M. Yurek, H. F. Taylor, and J. F. Weller, “35 $\mathrm{GHz}$ microwave signal generation with an injection-locked laser diode," Electronics Letters, vol. 21, no. 18, pp. 814-815, 1985.

[23] J. Yu, Z. Jia, L. Yi, Y. Su, G.-K. Chang, and T. Wang, "Optical millimeter-wave generation or up-conversion using external modulators," IEEE Photonics Technology Letters, vol. 18, no. 1, pp. 265-267, 2006.

[24] S. Pan and J. Yao, “Tunable subterahertz wave generation based on photonic frequency sextupling using a polarization modulator and a wavelength-fixed notch filter," IEEE Transactions on Microwave Theory and Techniques, vol. 58, no. 7, pp. 1967-1975, 2010.

[25] G. Qi, J. Yao, J. Seregelyi, S. Paquet, and C. Bélisle, "Optical generation and distribution of continuously tunable millimeterwave signals using an optical phase modulator," Journal of Lightwave Technology, vol. 23, no. 9, pp. 2687-2695, 2005.

[26] S. Chen, C. Zhao, Y. Li et al., "Broadband optical and microwave nonlinear response in topological insulator," Optical Materials Express, vol. 4, no. 4, pp. 587-596, 2014.

[27] F. Fan and M. Dagenais, "Optical generation of a megahertzlinewidth microwave signal using semiconductor lasers and a discriminator-aided phase-locked loop," IEEE Transactions on Microwave Theory and Techniques, vol. 45, no. 8, pp. 1296-1300.

[28] A. Hirata, M. Harada, K. Sato, and T. Nagatsuma, "Low-Cost Millimeter-Wave Photonic Techniques for Gigabit/s Wireless Link," IEICE Transactions on Electronics, vol. E86-C, no. 7, pp. 1123-1128, 2003.

[29] D. Wake, C. R. Lima, and P. A. Davies, "Optical generation of millimeter-wave signals for fiber-radio systems using a dual-mode DFB semiconductor laser," IEEE Transactions on Microwave Theory and Techniques, vol. 43, no. 9, pp. 2270-2276, 1995.

[30] H.-J. Song, N. Shimizu, T. Furuta, K. Suizu, H. Ito, and T. Nagatsuma, "Broadband-frequency-tunable sub-terahertz wave generation using an optical comb, AWGs, optical switches, and a uni-traveling carrier photodiode for spectroscopic applications," Journal of Lightwave Technology, vol. 26, no. 15, pp. 2521-2530, 2008.

[31] X. Chen, Z. Deng, and J. Yao, "Photonic generation of microwave signal using a dual-wavelength single-longitudinalmode fiber ring laser," IEEE Transactions on Microwave Theory and Techniques, vol. 54, no. 2, pp. 804-809, 2006.

[32] D. Chen, H. Fu, W. Liu, Y. Wei, and S. He, "Dual-wavelength single-longitudinal-mode erbium-doped fibre laser based on fibre Bragg grating pair and its application in microwave signal generation," Electronics Letters, vol. 44, no. 7, pp. 459-461, 2008.

[33] S. Hoffmann and M. R. Hofmann, "Generation of Terahertz radiation with two color semiconductor lasers," Laser and Photonics Reviews, vol. 1, no. 1, pp. 44-56, 2007.

[34] C. Tang, R. Li, Y. Shao et al., "Experimental demonstration for $40-\mathrm{km}$ fiber and $2-\mathrm{m}$ wireless transmission of $4-\mathrm{Gb} / \mathrm{s}$ OOK signals at $100-\mathrm{GHz}$ carrier," Chinese Optics Letters, vol. 11, no. 2, Article ID 020608, 2013.

[35] L. Ren, X. Qi, Y. Liu et al., "Large-scale production of ultrathin topological insulator bismuth telluride nanosheets by a hydrothermal intercalation and exfoliation route," Journal of Materials Chemistry, vol. 22, no. 11, pp. 4921-4926, 2012.

[36] S. Lu, C. Zhao, Y. Zou et al., "Third order nonlinear optical property of Bi2Se3," Optics Express, vol. 21, no. 2, pp. 2072-2082, 2013. 

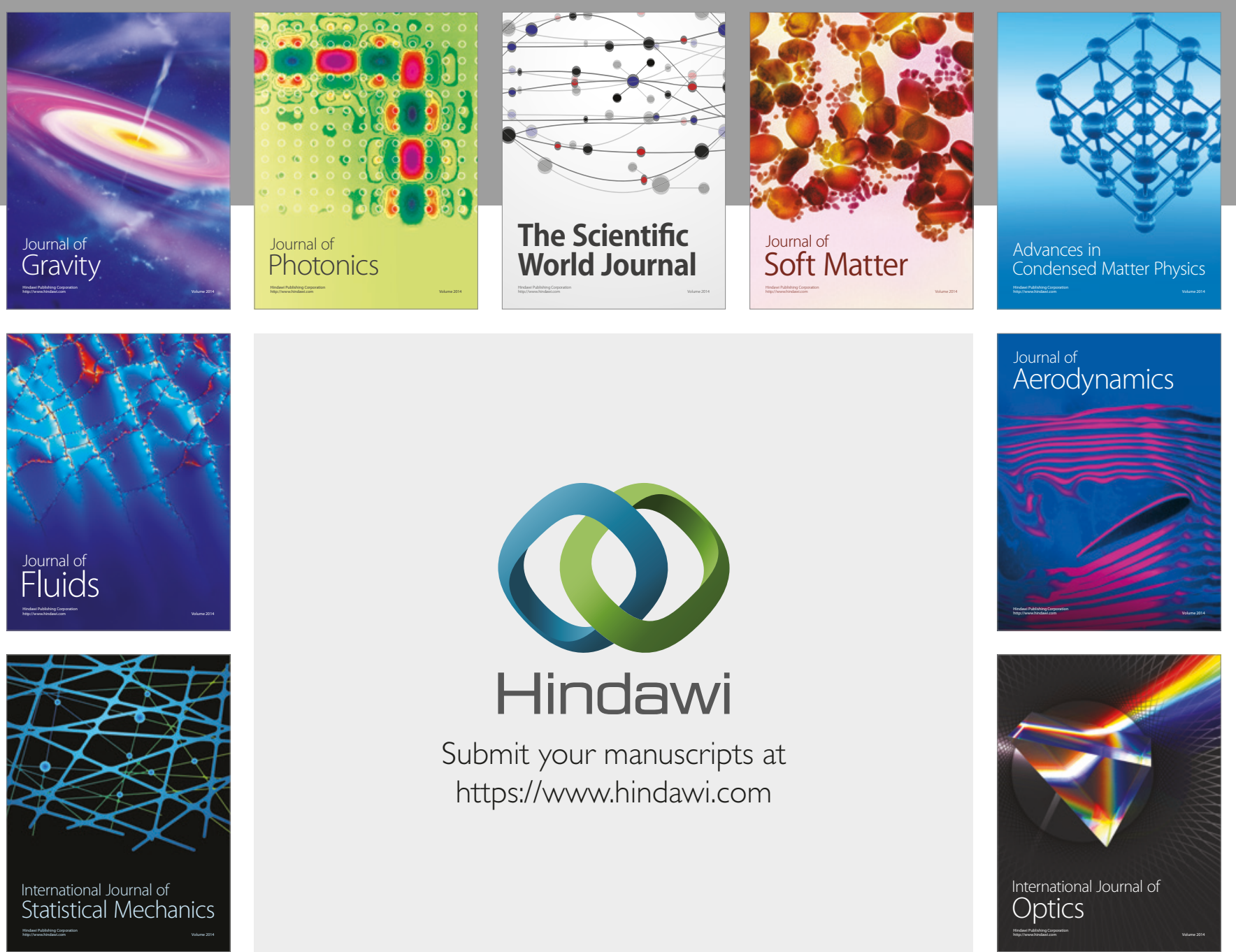

Submit your manuscripts at

https://www.hindawi.com
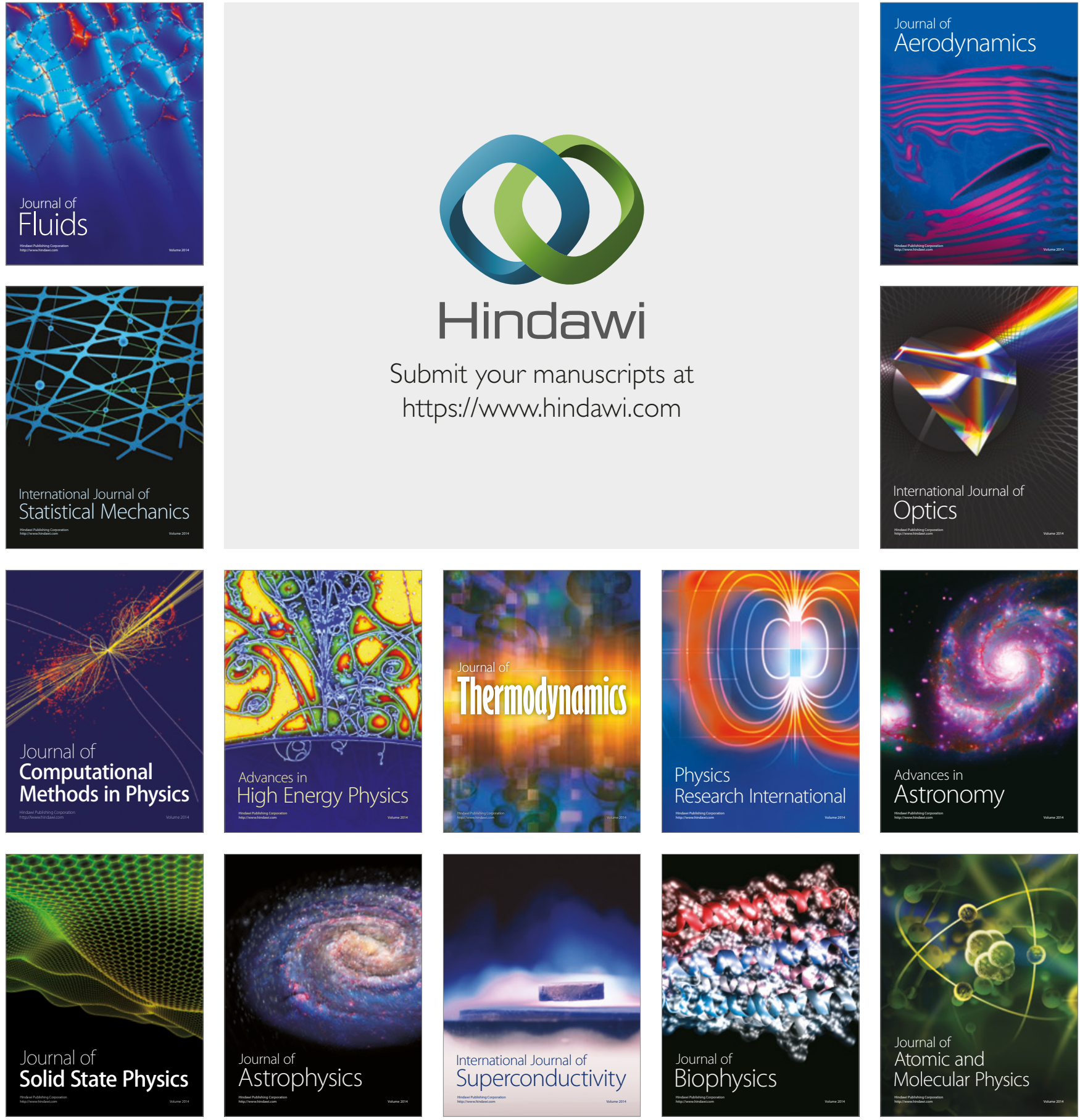